\title{
Hepatic Nodules Associated with an Inferior Mesentric Arteriovenous Malformation
}

\author{
Ken Takahashi ${ }^{1,2}$, Hiroshi Kashida ${ }^{1,3}$ and Masatoshi Kudo ${ }^{3}$
}

\begin{abstract}
Splanchnic arteriovenous malformation (AVM) is a rare condition in which patients present with portal hypertension, which thus causes bleeding varices and ascites. However, to our knowledge, hepatic nodules associated with splanchnic AVM have not yet been described. We herein first report the case of a 78-year-old man with inferior mesenteric AVM presenting with portal hypertension and multiple hepatic nodules dominantly supplied by the portal vein. This unique case not only extends the spectrum of hepatic nodules resulting from abnormal hepatic circulation, but also provides clues for better understanding the etiology of hepatic nodules.
\end{abstract}

Key words: splanchnic arteriovenous malformation, portal hypertension, hepatic nodule

(Intern Med 51: 2753-2755, 2012)

(DOI: 10.2169/internalmedicine.51.8395)

\section{Introduction}

With recent advances in imaging techniques, increasing numbers of benign hepatic nodules have been reported in non-cirrhotic patients. In such cases, abnormal hepatic circulation has been considered to be a possible etiological factor. Splanchnic arteriovenous malformation (AVM) is a rare but well-known cause of secondary portal hypertension (1). We herein report the first documented case of hepatic nodules associated with splanchnic AVM. The present case is quite unique in that the 'extrahepatic' abnormal circulation caused by splanchnic AVM induced 'intrahepatic' hemodynamic alterations that finally led to hepatic nodular formation. In this report, the imaging studies are presented and the possible etiology is discussed.

\section{Case Report}

A 78-year-old man with a recent history of progressive abdominal fullness was referred to our hospital for treatment of massive ascites and evaluation of multiple hepatic nodules demonstrated on ultrasound sonography (US). The pa- tient was not a regular alcohol drinker. The liver function tests results as well as other routine laboratory data, including the serum glucose and lipid levels, were all within normal limits. Viral markers for hepatitis B and C and serological markers of autoimmune disease were also negative.

Abdominal US showed massive ascites and the presence of multiple hepatic nodules in Couinaud's segment V and VI with homogenous parenchyma. Neither atrophic nor fatty changes were observed. On contrast-enhanced US, the nodules were clearly enhanced in the portal phase (Fig. 1). Unenhanced computed tomography (CT) showed multiple low attenuating areas corresponding to the findings observed on US. The lesions were hypoattenuating in the arterial phase and enhanced in the portal phase on dynamic CT (not shown). Angiography demonstrated a dilated inferior mesentric artery (IMA) supplying an AVM (Fig. 2A) thus leading to the early filling of the large inferior mesentric vein (IMV) draining into the portal vein (Fig. 2B). CT hepatic arteriogram (CTHA) clearly showed the nodules demonstrated on US and CT to be hypoattenuating lesions (Fig. 3A), while CT during arterioportogram (CTAP) showed the nodules to be hyperattenuating lesions (Fig. 3B). Fourteen days after admission, the patient passed massive melena and an emer-

\footnotetext{
${ }^{1}$ Department of Gastroenterology and Hepatology, Kobe City General Hospital, Japan, ${ }^{2}$ Department of Gastroenterology and Hepatology, Graduate School of Medicine, Kyoto University, Japan and ${ }^{3}$ Department of Gastroenterology and Hepatology, Kinki University School of Medicine, Japan

Received for publication June 17, 2012; Accepted for publication July 12, 2012

Correspondence to Dr. Ken Takahashi, takaken@kuhp.kyoto-u.ac.jp
} 


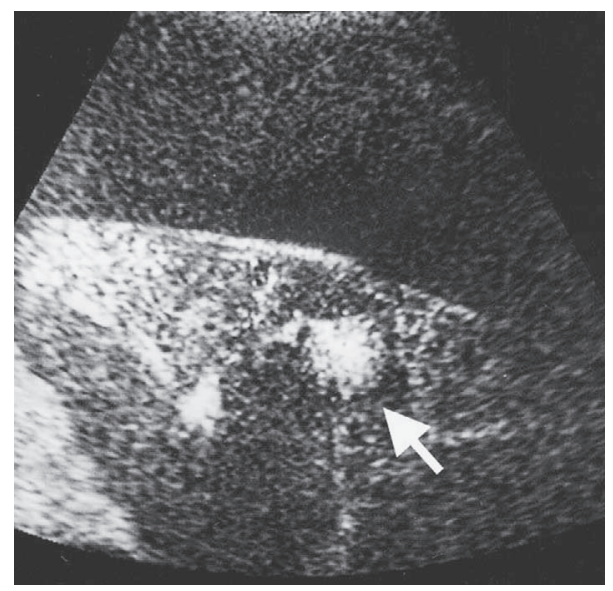

Figure 1. Portal-phase dynamic sonogram obtained 40 seconds after contrast medium injection revealing marked enhancement of the hepatic nodule in segment VI (arrow) on right intercostal scanning. Massive ascites is also demonstrated.

gency upper gastrointestinal endoscopy revealed bleeding esophageal varices that were successfully treated with an endoscopic variceal ligation technique. Colonoscopy also revealed rectal varices.

Since the patient had no signs of liver cirrhosis, we speculated that the increased portal flow due to the inferior mesentric AVM was the cause of the portal hypertension. The patient underwent resection of the AVM. The intraoperative findings revealed a normal appearance of the liver. The pathological analysis of the resected AVM revealed dilated, thick-walled vascular channels (both arterial and venous) primarily occupying the serosal surface of the sigmoid colon; the findings were consistent with those of an AVM. Postoperatively, the ascites and hepatic nodular lesions disappeared rapidly, as confirmed on both CT and US performed one month after surgery. A follow-up gastrointestinal endoscopy showed marked regression of the esophageal and rectal varices. The patient was discharged 42 days after surgery and has since been free from any symptoms.

\section{Discussion}

Splanchnic AVMs are rare conditions. In most cases, these lesions develop between hepatic or splenic arteries and the portal vein (1). AVMs involving the inferior mesenteric vessels are very rare (2). AVMs usually develop following ruptured aneurysms or occur secondary to surgical procedures or trauma (1). On the other hand, congenital AVMs have been reported in only a few cases (2). Based on the pathological analysis of surgical specimens and the lack of any history of abdominal surgery or trauma, the present case was considered to be congenital.

Splanchnic AVMs can cause secondary portal hypertension in non-cirrhotic patients, often leading to ascites and variceal formation (1). However, to our knowledge, this is the first documented case of hepatic nodules associated with

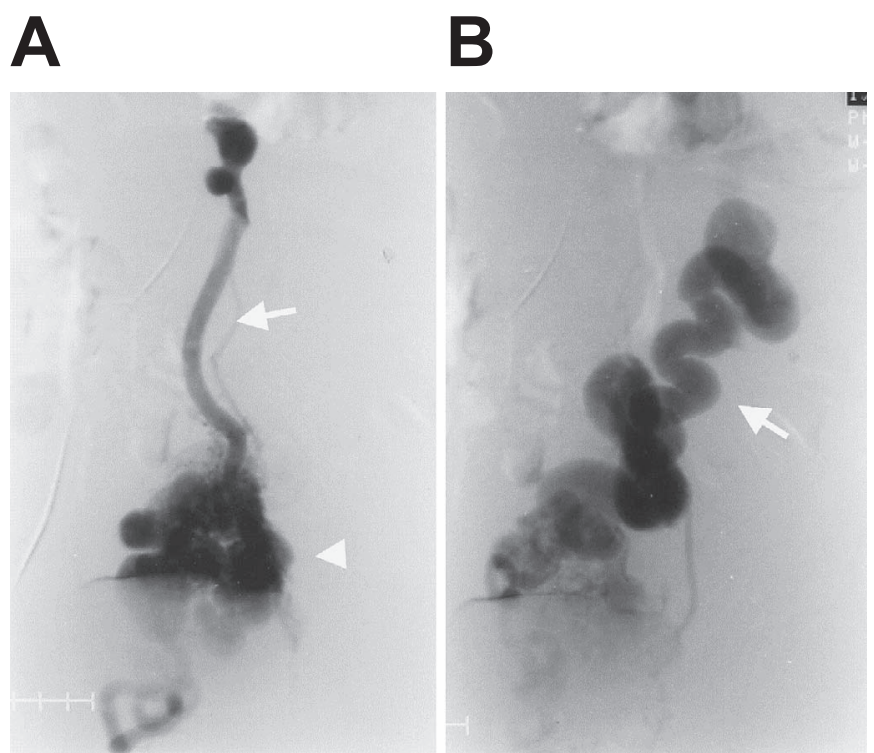

Figure 2. (A) Inferior mesentric arteriogram revealing an AVM (arrow head) supplied by a large IMA (arrow). (B) Inferior mesentric arteriogram obtained two seconds after panel A showing an early filling of IMV (arrow), indicating arterioportal shunting through the AVM.

splanchnic AVM. Unfortunately, owing to massive ascites, we could not obtain any biopsy samples of the nodules before treatment. However, the fact that the nodular lesions disappeared soon after the surgical removal of the AVM is direct evidence of the AVM's contribution to the formation of the hepatic nodules.

In recent years, reports of benign hepatic nodules in noncirrhotic livers have increased with advances in imaging techniques. In such cases, abnormal hepatic circulation has been considered as a possible etiological factor (3). Accordingly, hepatic nodules have been described in association with Budd-Chiari syndrome, a disorder resulting from the gross outflow blockage of hepatic veins with various causes, or congenital absence of the portal vein $(4,5)$. In addition, recently Ikeda et al. reported the presence of portal flowdominant hepatic nodules in a patient with idiopathic portal hypertension (6). The present case is quite unique in that the 'extrahepatic' abnormal circulation caused by the splanchnic AVM induced 'intrahepatic' hemodynamic alterations that finally led to hepatic nodular formation. Although the exact pathogenesis of the hepatic nodules in our case remains obscure, one possible explanation might be that the increased and arterialized portal blood flow distributing heterogenously caused focal hepatic injury and the reactive regeneration occurred as a process of repair of the damaged hepatic tissue. Starzl et al. reported that hormonal factors such as insulin, glucagon and epidermal growth factor in the mesentric vein might be important for hepatocyte function and structure and may play vital roles in hepatic regeneration (7). Therefore, an influx imbalance of such substances combined with uneven hepatic perfusion also contributes to the pathogenesis of nodular formation. 

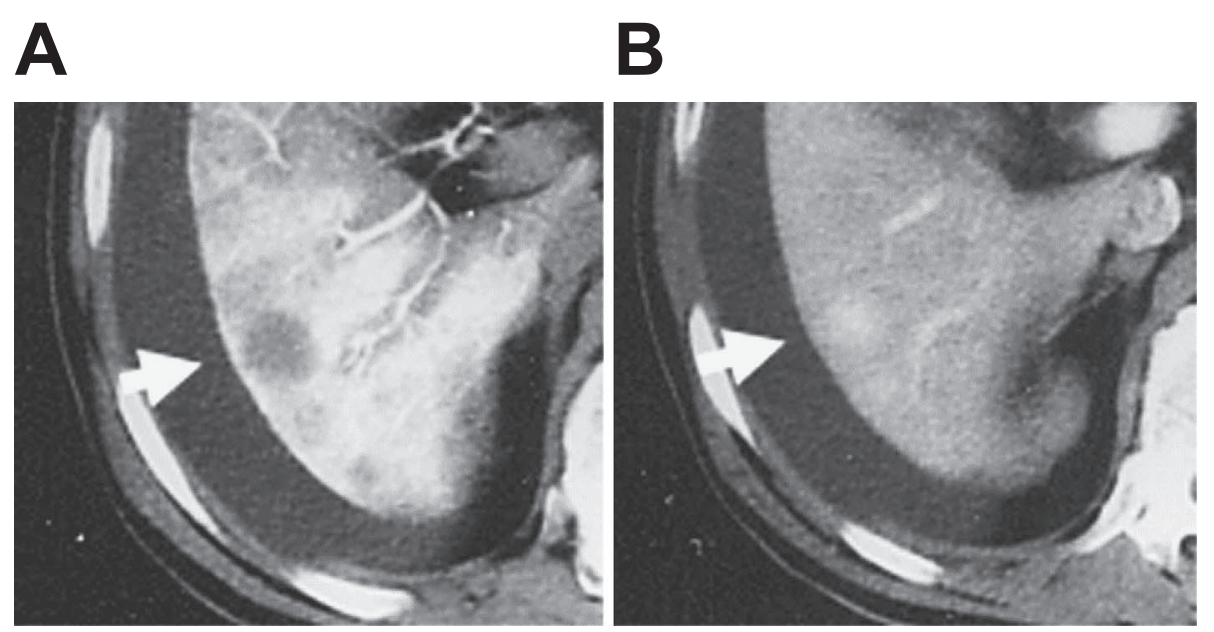

Figure 3. (A) CTHA revealing a hypoattenuating nodular lesion (arrow) in hepatic segment VI. (B) The same lesion is demonstrated as a hyperattenuating nodular lesion (arrow) on CTAP.

In conclusion, we herein presented a case of hepatic nodules associated with splanchnic AVM. This unique case not only extends the spectrum of hepatic nodules resulting from abnormal hepatic circulation, but also provides new insight for better understanding the etiology of hepatic nodules.

The authors state that they have no Conflict of Interest (COI).

\section{References}

1. Van Way CW, Crane JM, Riddell DH, Foster JH. Arteriovenous fistula in the portal circulation. Surgery 70: 876-890, 1971.

2. Baranda J, Pontes JM, Portela F, et al. Mesentric arteriovenous fistula causing portal hypertension and bleeding duodenal varices.
Eur J Gastroenterol Hepatol 8: 1223-1225, 1996.

3. Kondo F. Benign nodular hepatocellular lesions caused by abnormal hepatic circulation: etiological analysis and introduction of a new concept. J Gastroenterol Hepatol 16: 1319-1328, 2001.

4. Maetani $\mathrm{Y}$, Itoh $\mathrm{K}$, Egawa $\mathrm{H}$, et al. Benign hepatic nodules in Budd-Chiari Syndrome: radiologic-pathologic correlation with emphasis on the central scar. AJR 178: 869-875, 2002.

5. Tsuji K, Naoki K, Tachiyama Y, et al. A case of congenital absence of the portal vein. Hepatol Research 31: 43-47, 2005.

6. Ikeda A, Kita R, Nasu A, et al. Idiopathic portal hypertension with multiple hepatic hyperplastic nodules supplied by portal vein. Ann Hepatol 11: 572-573, 2012.

7. Starzl TE, Francavilla A, Halgrimson CG, et al. The origin, hormonal nature, and action of hepatotrophic substances in portal venous blood. Surg Gynecol Obstet 137: 179-199, 1973.

(C) 2012 The Japanese Society of Internal Medicine http://www.naika.or.jp/imonline/index.html 